\title{
A Case for Alpha-Lipoic Acid as an Alternative Treatment for Diabetic Polyneuropathy
}

\author{
Ngoc Nguyen ${ }^{1}$, Jody K. Takemoto ${ }^{1,2}$ \\ ${ }^{1}$ Ben and Maytee Fisch College of Pharmacy, The University of Texas at Tyler, Tyler, Texas. ${ }^{2}$ Department of Pharmaceutical \\ Sciences, Ben and Maytee Fisch College of Pharmacy, The University of Texas at Tyler, Tyler, Texas
}

Received, July 26, 2018; Accepted, August 20, 2018; Published, August 21, 2018.

\begin{abstract}
Purpose: The aim of this systematic review is to evaluate current evidence of alpha-lipoic acid (ALA) regarding efficacy, safety, and cost to accurately compare it with other diabetic polyneuropathy (DPN) treatments. The intention is to provide recommendations on future research and to promote utilization of ALA in the United States (US). Methods: A literature search was conducted on three databases: Scopus, PubMed, and Web of Science. The following criteria were used to select studies: (1) randomized controlled trials (RCTs) and open-label trials on ALA, (2) review articles and meta-analyses of RCTs on ALA, (3) study population consisting of patients with diabetes mellitus, peripheral neuropathic pain, and/or metabolic syndrome. Results: Twenty-five publications were selected including five RCTs and three open-label studies. Most clinical trials were conducted outside of the US. Current data provides evidence for the benefits of ALA in DPN treatment at a dose of $600 \mathrm{mg}$ per day, either intravenously (IV) or orally, for a duration of at least 3 weeks with minimal side effects. Conclusions: ALA demonstrates effectiveness in treating DPN through multiple mechanisms to modulate pathophysiology and control symptoms. In addition, ALA exhibits activity in weight management and insulin sensitivity. The use of ALA for DPN in the US is worth considering because commonly prescribed medications have unclear mechanisms, more pronounced adverse effects, and are more expensive than ALA. Further research needs to be conducted to assess long-term efficacy of ALA in US patients.
\end{abstract}

\section{INTRODUCTION}

Diabetes is among the most prevalent chronic diseases of the $21^{\text {st }}$ century, with an estimation of 30.3 million people living with diabetes $(1,2)$. The most common diabetic complication is diabetic polyneuropathy (DPN), which has a lifetime prevalence of approximately $50 \%$ in patients with diabetes (1). DPN significantly lowers quality of life as patients experience symptoms such as pain, abnormal sensation, muscle weakness, gait disturbances, and increased fall risk. The number of patients with diabetic neuropathy may exceed the estimated statistics due to unreported symptoms. This creates a burden on the health care system in the form of higher medical costs, lost productivity, premature mortality, and intangible costs due to reduced quality of life (3). The annual cost of diabetes in the US in 2017 was $\$ 327$ billion, increasing by $26 \%$ from 2012 due to increased prevalence and cost per person. It has been estimated that neurological complications, including DPN, comprised of $36 \%$ of this annual cost (3).

While the cause of DPN is multifactorial, it is largely attributed to oxidative stress from uncontrolled hyperglycemia $(4,7,8)$. Glucosemediated increase in reactive oxygen species (ROS) causes damage to the endothelial lining, including neural microvasculature (4). The pathways by which hyperglycemia causes damage to the vasculature is illustrated in figure 1.

Treatments for DPN include therapies that alter pathological pathways and those that reduce symptoms. Glucose control is the common diseasemodifying treatment by reducing hyperglycemiainduced damage. However, clinical trials in type 2 diabetes yielded inconsistent results, which emphasizes that many patients with type 2 diabetes develop DPN despite adequate glucose control (5). Therefore, pain management is the mainstay of therapy because it can significantly improve patients' quality of life. Nonetheless, the use of antidepressants, antiepileptics, or opioids are costly with modest efficacy and problematic adverse events such as sedation, nausea, anxiety, and cardiovascular complications or even addiction (6).

Corresponding Author: Jody K. Takemoto, The University of Texas at Tyler, Ben and Maytee Fisch College of Pharmacy, 3900 University Blvd. Tyler, TX; Email: jtakemoto@uttyler.edu 


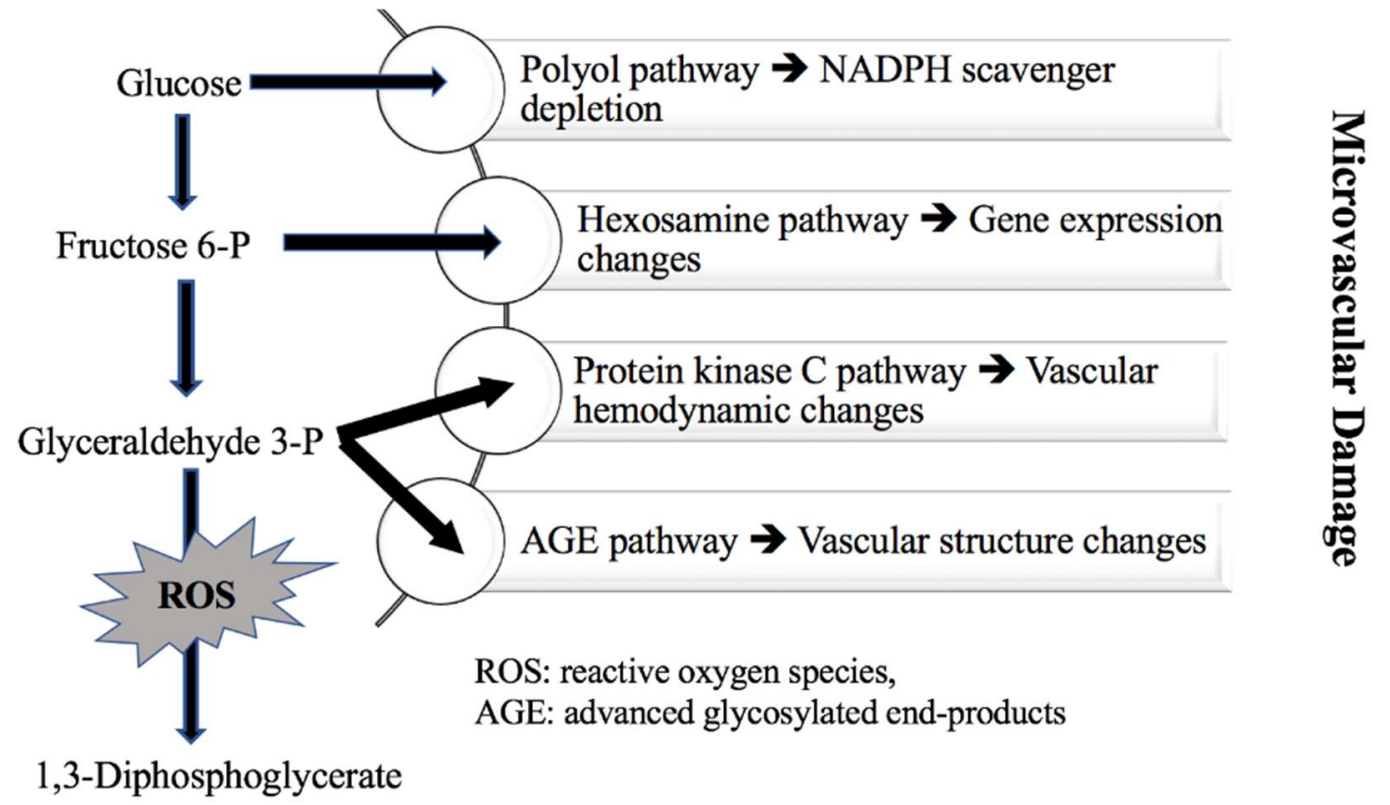

Figure 1. Mechanisms of hyperglycemia-induced microvascular damage
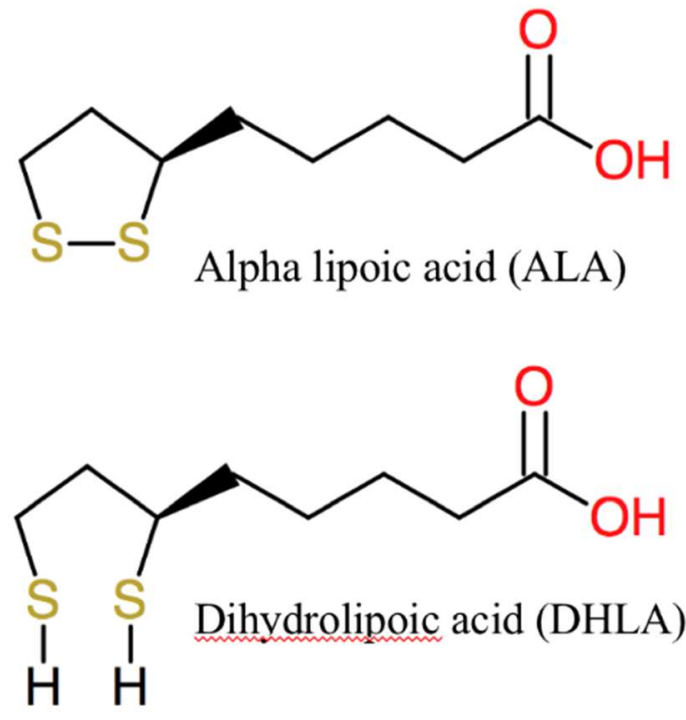

Figure 2. Chemical structures of lipoic acid

Alpha-lipoic acid (ALA), also called thioctic acid, provides both disease modulation and symptom control through various mechanisms. Figure 2 depicts the structures of oxidized (ALA) and reduced (DHLA) forms of ALA; both act as active antioxidants. In comparison with other antioxidants, ALA/DHLA have higher redox potential than that of glutathione, a natural antioxidant found in the body (7). They can also regenerate several antioxidants and are not destroyed while quenching free radicals like ascorbic acid. In addition, the two forms of thioctic acid can chelate redox-active metals. ALA is a potent chelator of divalent metal ions in vitro, while DHLA inhibits ascorbate oxidation by iron and coppers (7). Furthermore, ALA offers pain control through blocking T-typed calcium channel on nociceptive neurons (8). ALA is found in low amounts in vegetables such as spinach, broccoli, 
tomatoes, and animal tissues with the highest concentration found in the kidneys, heart, and liver (8). In the US, it is available as over-the-counter and is used for weight loss and diabetes supplement.

An earlier meta-analysis of randomized controlled trials (RCTs) on the use of ALA at 600 $\mathrm{mg}$ /day intravenously in patients with diabetes and neuropathic pain concluded that three weeks of treatment resulted in significant pain reduction (9). Another meta-analysis of four RCTs on ALA at various doses of oral and intravenous administrations confirmed the effectiveness of intravenous ALA but stated that oral ALA had unclear clinical relevance (4). Many other review articles on ALA focus on mechanisms of cellular action instead of providing clinical relevance. While some literature already outlined the potential application of ALA; to our best knowledge, none have addressed the specific clinical application of this supplement in US population.

Even though ALA demonstrates favorable mechanisms in treating DPN, its utilization in the US is limited. This is partly due to the fact that most literature on ALA are foreign, with the majority of trials conducted in Germany. In Germany, ALA is approved for the treatment of diabetic neuropathy pain and is on many insurance formularies (14-16). In the US, ALA is sold as a supplement over-thecounter and therefore, does not have an indication to be covered by insurances.

In order to examine all current evidence, this review article includes open label trials along with RCTs using both intravenous and oral forms of ALA. These studies are also not limited to one geographical location. Furthermore, this review not only looks at the effects of ALA on neuropathic pain, but also on metabolic component of diabetes such as weight change and insulin sensitivity. Cost minimization is also discussed to assist in the choice between ALA and conventional neuropathic pain management.

The aim of this systematic review is to evaluate current evidence of ALA efficacy and its safety profile in obese and diabetic patients to accurately compare it with other DPN treatments. This review article brings attention to ALA as a cost-effective alternative treatment. We also discuss other therapeutic benefits of ALA in weight management and potential use in the obstetric population. The intention is to provide recommendations on future research and to promote utilization of ALA in the US.

\section{MATERIAL AND METHODS}

\section{Literature Search}

A search was conducted on three databases: Scopus, MEDLINE using the search engine PubMed, and Web of Science. Specifiers were added to filter for the newest available evidence. The search details are described in figure 3.

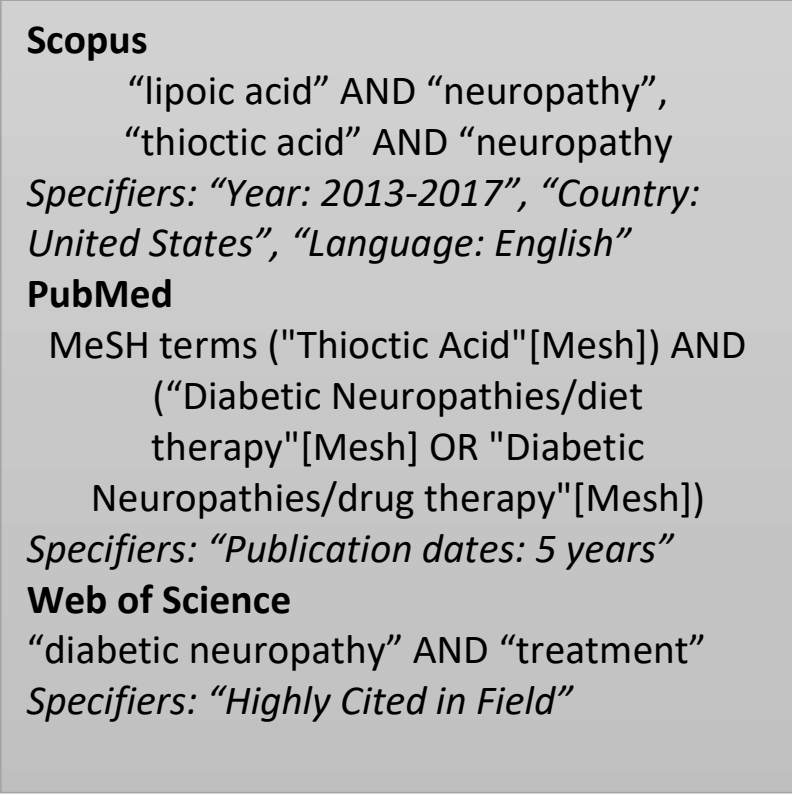

Figure 3. Search strategies

\section{Study Selection}

The following criteria were used to select studies: (1) RCTs and open-label trials on ALA, (2) review articles and meta-analyses of RCTs on ALA, (3) study population consisting of patients with diabetes mellitus, peripheral neuropathic pain, and/or metabolic syndrome. Language was a restriction in the search strategy; only articles in English were selected. Specifiers were also added to select publications from the last five years. From Web of Science, only articles highly cited in the field were chosen. The reference lists of identified manuscripts were reviewed for potentially eligible studies. Unpublished data were excluded from this review.

\section{Outcome Measure}

The primary outcome measures related to diabetic neuropathy in this review article were total symptom score (TSS), neuropathy impairment score (NIS) and analgesic use. Other outcomes related to diabetes 
included insulin sensitivity, glucose effectiveness, body weight, and contrast sensitivity in retinopathy.

\section{RESULTS}

The search in PubMed using MeSH terms, Web of Science, and Scopus yielded 99, 5990, and 1454 results, respectively. While PubMed and Scopus databases were used to locate literature on ALA therapy, Web of Science was utilized to search for other treatments of diabetic neuropathy. The use of specifiers to refine results yielded a total of 212 articles. As the focus of the review was benefits of ALA over conventional treatments for DPN, 11 trials (5 RCTs), 3 animal studies, and 14 review articles and meta-analyses were found to meet aforementioned criteria. However, 3 articles were eliminated due to unavailability in English. Complete article reviews narrowed the search down to 25 publications.

Pertaining to route of administration of ALA in RTCs, all but one study used oral form of ALA with duration of treatment ranged from 3 to 20 weeks. The primary outcome from 5 trials were DPN symptom reduction, which was measured using various tools such as total symptom score (TSS), neuropathy impairment score (NIS), or a composite score. These tools were primarily developed for the evaluation of therapeutic efficacy or the longitudinal assessment of polyneuropathy (10). Three studies evaluated the indirect effects of ALA in DPN patients on body weight change, diabetic retinopathy, and through insulin sensitivity and glucose effectiveness. Most of the clinical trials that met our criteria were conducted outside of the USA except one. Table 1 provides a summary of clinical trials that met the criteria. Overall, the results are in alignment with the hypothesis of ALA beneficial application in the treatment of DPN.

\section{DISCUSSION}

Results from selected studies provide evidence for the benefits of ALA in treatment of DPN at a dose of $600 \mathrm{mg}$ per day, either intravenously or orally, for a duration of at least 3 weeks. Dosages above $600 \mathrm{mg}$ once a day did not show any statistical and clinical difference (e.g. stabbing pain, burning pain, paresthesia, asleep numbness of the feet) in comparison to the control arms (15). Most studies assessed ALA effect in DPN using total symptom control (TSS) score, which is a validated tool to evaluate therapeutic efficacy in neuropathy sensory symptoms. TSS is a summation of presence, severity, and duration of the 4 main symptoms: lancinating/stabbing pain, burning pain, paresthesia, and asleep numbness (14). The majority of RCTs on ALA in DPN treatment were conducted by a German research group in studies called NATHAN 1, SYDNEY, SYDNEY 2 ((14-16), Table 1). The SYDNEY trial in 2003 showed statistical improvement in TSS of $600 \mathrm{mg}$ IV ALA for 5 days per week for 3 weeks (15). Consequently, SYDNEY 2 compared different doses of oral ALA administrations and found no statistical differences in TSS score among $600 \mathrm{mg}, 1,200 \mathrm{mg}$ and 1,800 mg doses (14). Lastly, NATHAN 1 studied the effect of chronic ALA administration in 4 years and found that ALA is associated with neuropathic improvements but not nerve conduction, hence no difference in the composite endpoint was found compared to placebo. This trial also concluded that long-term treatment of ALA was well-tolerated (14). In these studies, improvements in neuropathy symptoms were seen after week 1 regarding neuropathic, numbness and paresthesia. The rapid improvement of various DPN symptoms is hypothesized to occur due to enhanced nerve blood flow mediated by the antioxidant action of the drug (9). This further elucidates ALA benefits as a potent antioxidant in DPN treatment. While providing valuable data on clinical application of ALA, these studies are subject to bias as they were conducted in multi-center European locations and were funded by VIATRIS, the manufacturer of ALA in Germany.

Effects of ALA on metabolism is demonstrated in a 2016 RCT conducted in China (Table 1). Li et al. evaluated the effect of an 8-week, once daily oral ALA at a dose of $1,200 \mathrm{mg} /$ day on weight reduction, waist circumference, lipid profile, leptin levels and adverse events (11). The study found that the treatment resulted in a slight but significant reduction in both waist circumference and body weight; however, no statistical significance was detected for other parameters, i.e., lipid profile, leptin levels, and adverse events. These findings can be attributed to small sample size of 116 patients as a trend towards improved lipid profile and a reduction in leptin levels were observed in the treatment group but failed to reach statistical significance. Common side effects of ALA included rash and nausea, which were predictable and manageable. Importantly, the trial identified that the ALA dose for neuropathic pain $(600 \mathrm{mg} /$ day $)$ was 
insufficient to cause weight reduction in obese patients. Some limitations of this trial include small sample size and short study duration. Also, the study was conducted on Chinese subjects, who might have different metabolic characteristics along with variations in diet and lifestyle from the US population. It is thought that the underlying cause of diabetic neuropathy is due to poor glycemic control which is mediated by the metabolic component of the disease; therefore, the findings suggest diseasemodifying properties of ALA. The results concord with the hypothesis about the safety and efficacy of ALA as a therapeutic option.

The pathophysiology of neuropathy and retinopathy in diabetes share the same component of microvascular damage due to oxidative stress. In a randomized, placebo-controlled study, Gebka et al demonstrated the benefit of oral ALA at a dose of $300 \mathrm{mg}$ daily for 3 months in preventing loss of vision in type 1 diabetes mellitus (T1DM) patients and improving it in T2DM patients (13). Concurrently, vision impairment measured by contrast sensitivity in both T1DM and T2DM patients without ALA supplementation were observed. The authors also suggested that in T2DM, ALA improves insulin sensitivity in addition to being a potent antioxidant, corroborating ALA ability to modify the pathophysiology of hyperglycemia-induced polyneuropathy by improving glycemic control.

Evidence from the 3 open-label studies from Table 1 also demonstrated statistical significance of ALA therapies, even though less rigorous designs and placebo effect might play a role in findings $(12,17,18)$. Garcia-Alcala et al assessed the difference in TTS between the ALA continuation and ALA withdrawal in patients who initially received $1800 \mathrm{mg} /$ day of ALA (orally, thrice a day) for 4 weeks. During the initial 4-week phase, the TSS was reduced in responders by $60 \%(\mathrm{p}<0.05)$, and it was further reduced in the continuation group by $32 \%(p<0.05)$. Similarly, the use of analgesics for neuropathic pain was reduced by $50 \%$ in the treatment arm (12). In addition, ALA, at a dose of $600 \mathrm{mg} /$ day orally for 8 weeks, demonstrated efficacy in reducing polyneuropathy TTS of at least $30 \%$ in another study conducted in South Korea by Halm et al (17). The findings from these two trials are in alignment with the hypothesis that ALA improves symptom control in polyneuropathy, specifically, a reduction in pain, as evidence by the reduction in TSS. Lastly, Konrad et al., showed that
ALA at $600 \mathrm{mg}$ orally twice a day for 4 weeks prevented hyperglycemia-induced increases of serum lactate and pyruvate in T2DM through aerobic glucose oxidation in the mitochondria. This discovery further demonstrates the efficacy of ALA in reducing damage caused by hyperglycemia at the molecular level (18).

Current guideline-recommended therapies for diabetic neuropathy are symptom-relieving as opposed to disease-modifying. With the exception of glycemic control, there is insufficient data for therapies that can prevent the progression of DPN. However, it has been demonstrated that ALA provides both disease modulation and symptom control by acting as an antioxidant and T-typed calcium channel blocker. According to American Diabetes Association report in 2017, patients with diabetes spent $\$ 71,235 /$ year on prescription medications other than insulin (3). This cost is likely a financial burden especially to the uninsured, underinsured, and those who are living on a limited income. Therefore, alternatives to provide symptomatic relief are necessary. Per the International Diabetes Federation (IDF) 2017 report, an average diabetic in Germany (where ALA is approved as a treatment) spent $\$ 6,235 /$ year while one in the US spent $\$ 12,135$ year with approximately $32 \%$ of the annual expenditure on diabetic medications other than insulin $(3,19)$. In the United States, the cost of using ALA at the dose of 600 $\mathrm{mg} /$ day starts at $\$ 10 /$ month while guideline-directed therapies such as gabapentin and duloxetine can cost up to $\$ 500 /$ month without insurance. Pertaining to the side effect profiles, the anticonvulsants and antidepressants can cause neurologic side effects ranging from somnolence, dizziness and headache to fall risk and serotonin syndrome. In a retrospective study by Ruessmann, patients were randomized into taking gabapentin or placebo after finishing a 5-year course of ALA at $600 \mathrm{mg} / \mathrm{day}$. It was found that in gabapentin group, $45 \%$ had to stop taking the drug due to intolerable side effects (somnolence, dizziness, vertigo, falls); $55 \%$ did not respond to gabapentin at doses up to $2,400 \mathrm{mg} /$ day, leading to the use of alternative or combination treatment. The total cost of gabapentin treatment is higher compared to that of ALA due to drug cost and increased doctor visits (20). An extensive review on preventing and treating DPN from Johns Hopkins University is also in agreement with Ruessmann's findings. The review pointed out that in general, all current pharmacologic treatments for DPN have 
substantial risks of adverse effects, which are of particular concern in older patients with diabetes. In particular, duloxetine had high rates of dropouts due to adverse effects, with rates of 17 to 20 percent in most study arms. Although studies on ALA provided lower strength of evidence, given the limitations of other pharmacologic approaches, ALA could be of particular value (21). These findings are in alignment with the hypothesis of favorable cost and tolerability of ALA compared with other DPN treatment. While there is no established maximum dose of ALA in humans, the use of ALA in trials has demonstrated a tolerable safety profile, with gastrointestinal disturbances (nausea, stomach upset) being the most common side effects. In fact, while most agents are contraindicated in pregnant women, ALA can potentially provide benefits for the obstetric population (22). Even though no clinal trials of ALA have included this special population, in vitro studies demonstrate ALA's positive effects in preventing the weakening of fetal membranes. Animal studies showed protective effects of ALA on fetuses whose mother are exposed to ROS from diabetes, alcohol and dioxin (22-25).

The promising findings of this review article on the role of ALA in DPN treatment should be interpreted in light of some limitations. Firstly, diabetic neuropathy is a chronic condition which likely requires the use of long-term pharmacotherapy; however, most studies were conducted over a short-term period, with the longest being 20 weeks (12). This necessitates more future studies on the chronic effectiveness and safety of alpha-lipoic acid. Secondly, the trials referenced in this article used different scales to measure their outcomes. A lack of a standardized tool can lead to misinterpretation of results. Thirdly, despite the effort to locate studies in the US, most studies found were conducted in foreign countries, which makes it problematic to apply their external validity to the US patient population. Finally, in the US, ALA is sold as dietary supplement from different manufacturers and does not fall under FDA strict regulations for quality control. Therefore, the purity of over-thecounter ALA is a factor to consider when making recommendations.

Nonetheless, data from heterogeneous studies all demonstrate ALA benefits in diabetic polyneuropathy in particular and metabolic disorder in general. ALA stands out as a therapeutic option in the treatment of DPN due to its favorable safety profile and cost-effectiveness along with its diseasemodifying and symptom-controlling mechanisms.

\section{CONCLUSION}

ALA demonstrates effectiveness in treating DPN through multiple mechanisms such as chelating metal ions, inhibiting reactive oxygen species, and blocking T-type calcium channels, which leads to improving nerve function and neuropathic pain. Studies analyzing the effects of ALA have shown significant reduction of neuropathy symptoms in diabetic patients. In addition, ALA exhibits activity in weight management and insulin sensitivity. The findings of this review article are consistent with previous findings about the benefit of ALA in diabetic patients. It has been approved as a DPN treatment in Germany for decades. In the US, ALA gained popularity as a weight loss and diabetic supplement. As commonly prescribed medications have unclear mechanisms of action and more pronounced adverse effects compared to ALA, it is appropriate to consider ALA as a treatment option for DPN. This article adds a cost minimization perspective which favors ALA over other therapies. Therefore, the findings support recommendations of further investigations of ALA as an alternative therapy in the US. The prospective advantage of this therapy might generate the need to develop ALA as a drug under FDA's Good Manufacturing Practice in order to maintain its quality consistently.

\section{ACKNOWLEDGEMENT}

The authors of this review article have no conflicts of interest to declare. No sources of funding were used to assist in the preparation of this manuscript. We acknowledge the help of Ms. Rebecca Fernandez for literature support.

\section{REFERENCES}

1. Juster-Switlyk K, Smith AG. Updates in diabetic peripheral neuropathy. [version 1; referees: 3 approved]. 2016 Apr 25;5.

2. U.S. Dept of Health and Human Services; C for DC and P. Centers for Disease Control and Prevention. National Diabetes Statistics Report, 2017. [Internet]. 2017 [cited 2017 Sep 11]. Available from: https://www.cdc.gov/diabetes/pdfs/data/statistics/nat ional-diabetes-statistics-report.pdf

3. American Diabetes Association. Economic costs of diabetes in the U.S. in 2017. Diabetes Care. 2018 Mar 
22;41(5):917-28.

4. Mijnhout GS, Kollen BJ, Alkhalaf A, Kleefstra N, Bilo HJG. Alpha lipoic Acid for symptomatic peripheral neuropathy in patients with diabetes: a meta-analysis of randomized controlled trials. Int $\mathbf{J}$ Endocrinol. 2012 Jan 26;2012:456279.

5. Pop-Busui R, Boulton AJM, Feldman EL, Bril V, Freeman R, Malik RA, et al. Diabetic neuropathy: A position statement by the american diabetes association. Diabetes Care. 2017;40(1):136-54.

6. Finnerup NB, Attal N, Haroutounian S, McNicol E, Baron R, Dworkin RH, et al. Pharmacotherapy for neuropathic pain in adults: a systematic review and meta-analysis. Lancet Neurol. 2015 Feb;14(2):16273.

7. Rochette L, Ghibu S, Richard C, Zeller M, Cottin Y, Vergely C. Direct and indirect antioxidant properties of $\alpha$-lipoic acid and therapeutic potential. Mol Nutr Food Res. 2013 Jan;57(1):114-25.

8. Lee WY, Orestes P, Latham J, Naik AK, Nelson MT, Vitko I, et al. Molecular mechanisms of lipoic acid modulation of T-type calcium channels in pain pathway. J Neurosci. 2009 Jul 29;29(30):9500-9.

9. Ziegler D, Nowak H, Kempler P, Vargha P, Low PA. Treatment of symptomatic diabetic polyneuropathy with the antioxidant alpha-lipoic acid: a metaanalysis. Diabet Med. 2004 Feb;21(2):114-21.

10. Yang Z, Chen R, Zhang Y, Huang Y, Hong T, Sun F, et al. Scoring systems to screen for diabetic peripheral neuropathy. Cochrane Database Syst Rev. 2014 Mar 5 ;

11. Li N, Yan W, Hu X, Huang Y, Wang F, Zhang W, et al. Effects of oral $\alpha$-lipoic acid administration on body weight in overweight or obese subjects: a crossover randomized, double-blind, placebocontrolled trial. Clin Endocrinol (Oxf). 2017 May;86(5):680-7.

12. Garcia-Alcala H, Santos Vichido CI, Islas Macedo S, Genestier-Tamborero CN, Minutti-Palacios M, Hirales Tamez O, et al. Treatment with $\alpha$-Lipoic Acid over 16 Weeks in Type 2 Diabetic Patients with Symptomatic Polyneuropathy Who Responded to Initial 4-Week High-Dose Loading. J Diabetes Res. 2015 Aug 4;2015:189857.

13. Gębka A, Serkies-Minuth E, Raczyńska D. Effect of the administration of alpha-lipoic acid on contrast sensitivity in patients with type 1 and type 2 diabetes. Mediators Inflamm. 2014 Feb 10;2014:131538.

14. Ziegler D, Low PA, Litchy WJ, Boulton AJM, Vinik AI, Freeman R, et al. Efficacy and safety of antioxidant treatment with $\alpha$-lipoic acid over 4 years in diabetic polyneuropathy: the NATHAN 1 trial. Diabetes Care. 2011 Sep;34(9):2054-60.

15. Ziegler D, Ametov A, Barinov A, Dyck PJ, Gurieva
I, Low PA, et al. Oral treatment with alpha-lipoic acid improves symptomatic diabetic polyneuropathy: the SYDNEY 2 trial. Diabetes Care. 2006 Nov;29(11):2365-70.

16. Ametov AS, Barinov A, Dyck PJ, Hermann R, Kozlova N, Litchy WJ, et al. The sensory symptoms of diabetic polyneuropathy are improved with alphalipoic acid: the SYDNEY trial. Diabetes Care. 2003 Mar;26(3):770-6.

17. Hahm JR, Kim B-J, Kim K-W. Clinical experience with thioctacid (thioctic acid) in the treatment of distal symmetric polyneuropathy in Korean diabetic patients. J Diabetes Complicat. 2004 Apr;18(2):7985.

18. Konrad T, Vicini P, Kusterer K, Höflich A, Assadkhani A, Böhles HJ, et al. alpha-Lipoic acid treatment decreases serum lactate and pyruvate concentrations and improves glucose effectiveness in lean and obese patients with type 2 diabetes. Diabetes Care. 1999 Feb;22(2):280-7.

19. IDF diabetes atlas - Home [Internet]. [cited 2018 Jun 17]. Available from: http://www.diabetesatlas.org/

20. Ruessmann H-J, German Society of out patient diabetes centres AND (Arbeitsgemeinschaft niedergelassener diabetologisch tätiger Arzte e.V.). Switching from pathogenetic treatment with alphalipoic acid to gabapentin and other analgesics in painful diabetic neuropathy: a real-world study in outpatients. J Diabetes Complicat. 2009 Jun;23(3):174-7.

21. Dy SM, Bennett WL, Sharma R, Zhang A, Waldfogel JM, Nesbit SA, et al. Preventing complications and treating symptoms of diabetic peripheral neuropathy. Rockville (MD): Agency for Healthcare Research and Quality (US); 2017.

22. Costantino M, Guaraldi C, Costantino D, De Grazia S, Unfer V. Peripheral neuropathy in obstetrics: efficacy and safety of $\alpha$-lipoic acid supplementation. Eur Rev Med Pharmacol Sci. 2014;18(18):2766-71.

23. Di Tucci C, Di Feliciantonio M, Vena F, Capone C, Schiavi MC, Pietrangeli D, et al. Alpha lipoic acid in obstetrics and gynecology. Gynecol Endocrinol. 2018 May $4 ; 1-5$.

24. Al Ghafli MHM, Padmanabhan R, Kataya HH, Berg B. Effects of a-lipoic acid supplementation on maternal diabetes-induced growth retardation and congenital anomalies in rat fetuses. Mol Cell Biochem. 2004 Jun;261(1):123-35.

25. Koga $T$, Ishida $T$, Takeda $T$, Ishii $Y$, Uchi $H$, Tsukimori $\mathrm{K}$, et al. Restoration of dioxin-induced damage to fetal steroidogenesis and gonadotropin formation by maternal co-treatment with $\alpha$-lipoic acid. PLoS One. 2012 Jul 20;7(7):e40322. 
J Pharm Pharm Sci (www.cspsCanada.org) 21, 192s - 199s, 2018

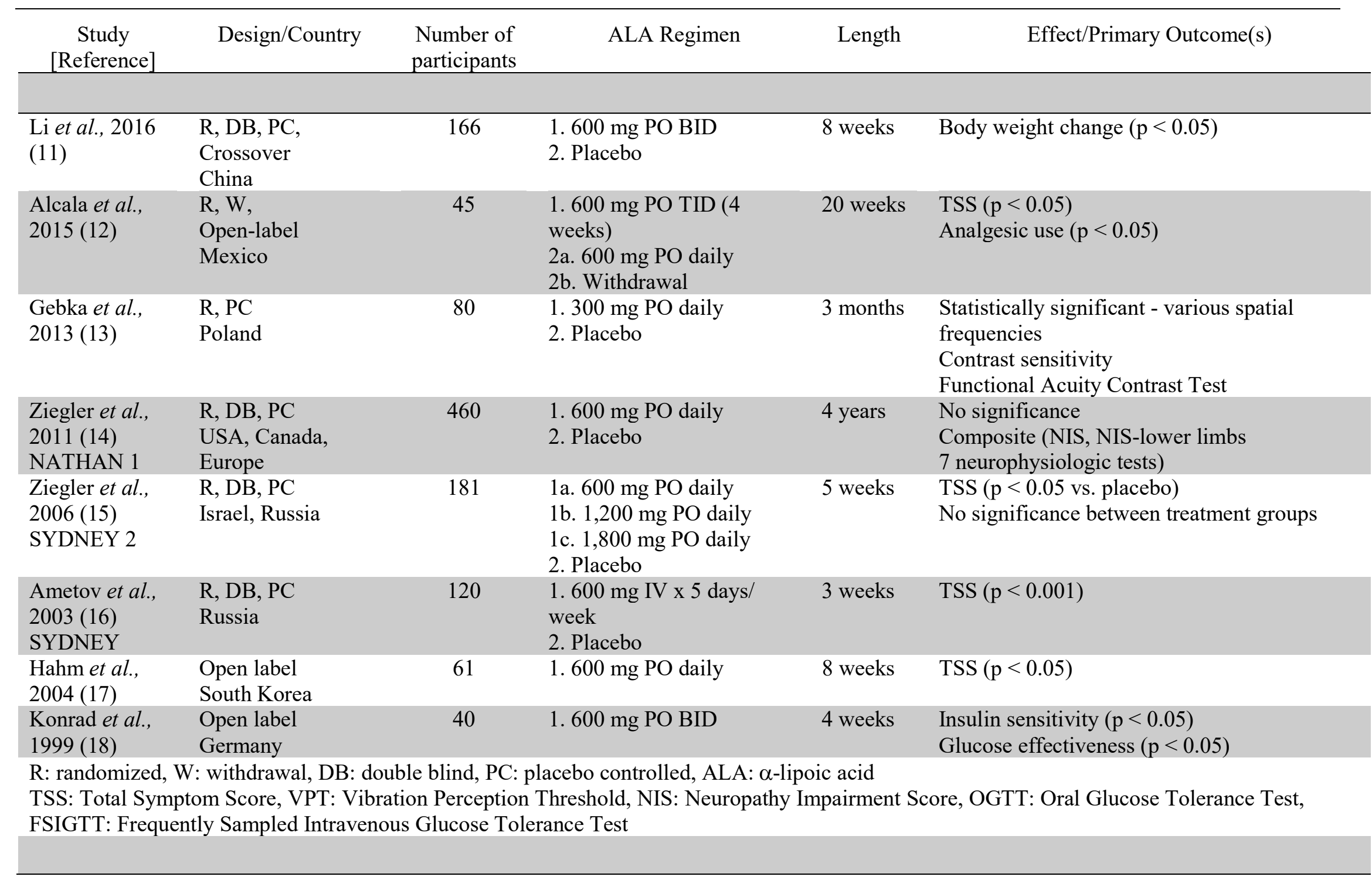

Table 1. Summary of clinical trials: a-lipoic acid in obese and/or diabetic patients 